\title{
Study on the Wide Gap Dielectric Barrier Discharge Device
}

\author{
Gaofeng Wang \\ School of Information Engineering, Zhengzhou University, Zhengzhou 450001, China \\ 932167312@qq.com
}

Keywords: DBD; Wide air gap; Plasma body; Modeling and simulation

\begin{abstract}
A high frequency and high voltage power supply for wide air gap dielectric barrier discharge (DBD) is introduced, and the application of dielectric barrier discharge device in industrial waste gas treatment is also discussed. The front stage of the power supply adopts the full bridge inverter technology, the rear stage adopts the high frequency transformer and the load series resonance, and frequency tracking technology, so that the front and back frequency matching, to achieve the maximum power output. This topic through the principle design, modeling and simulation, physical testing, the final realization of power and frequency are continuously adjustable wide air gap dielectric barrier discharge.
\end{abstract}

\section{Introduction}

With the development of economy, more and more industrial waste gas produced, these gases often contain sulfides, nitrides, and toxic organic compounds [1]. These gases have a great pollution to the environment caused by haze, acid rain, greenhouse effect and so on a series of environmental problems, the greater harm, cause bronchitis, asthma, emphysema and pulmonary heart disease and other respiratory diseases [2]. There are many methods to deal with the waste gas, such as activated carbon adsorption, catalytic combustion, catalytic oxidation, acid base neutralization, dielectric barrier discharge (DBD). The dielectric barrier discharge (DBD) method has the advantages of single particle production, relatively low energy consumption and no pollution once again. The dielectric barrier discharge (DBD) treatment of industrial waste gas has been widely used in developed countries, especially Germany and the United States. But at present, there is a big gap between China and foreign countries in the process of waste gas treatment. Mainly in the single yield low, heavy volume, frequency power supply efficiency problem low in dielectric barrier discharge (DBD) device [3].In dielectric barrier discharge (DBD) device, the power supply is one of the key components of the dielectric barrier discharge device, intelligent high power high frequency and power of the dielectric barrier discharge (DBD) is the direction of development.

\section{Design of Dielectric Barrier Discharge}

Principle of Dielectric Barrier Discharge. Dielectric barrier discharge (DBD) is a kind of method to generate plasma by inserting the dielectric into the discharge space [4]. The discharge space plasma produced by dielectric barrier discharge (DBD) in high pressure and a wide range of frequencies, usually working pressure is $10 \sim 10000 \mathrm{~Pa}$. power frequency from $50 \mathrm{~Hz}$ to $1 \mathrm{MHz}$. electrode junctions have various forms the full diversity. A working gas between the two electrode, and one or two electrode with insulation cover, when applied between two electrodes AC voltage is high enough, the gas between the two electrodes can be produced breakdown discharge, which produce dielectric barrier discharge.

Dielectric barrier discharge (DBD) equivalent diagram as shown in Fig. 1, the high frequency AC power to the discharge device, which is composed of an insulating medium discharge device for the upper and lower electrodes and intermediate. When the voltage applied to the discharge device is lower than the discharge voltage [5], power supply device for loading equivalent to a capacitor. When alternating current increases to the minimum discharge over voltage discharge device, discharge device to start work, discharge gap gradually stabilized, the voltage across the discharge gap with the increase of alternating current will not change, this device is equivalent to the 
discharge gap capacitance and dielectric equivalent capacitance in series, wherein the discharge gap capacitance voltage is a fixed value.

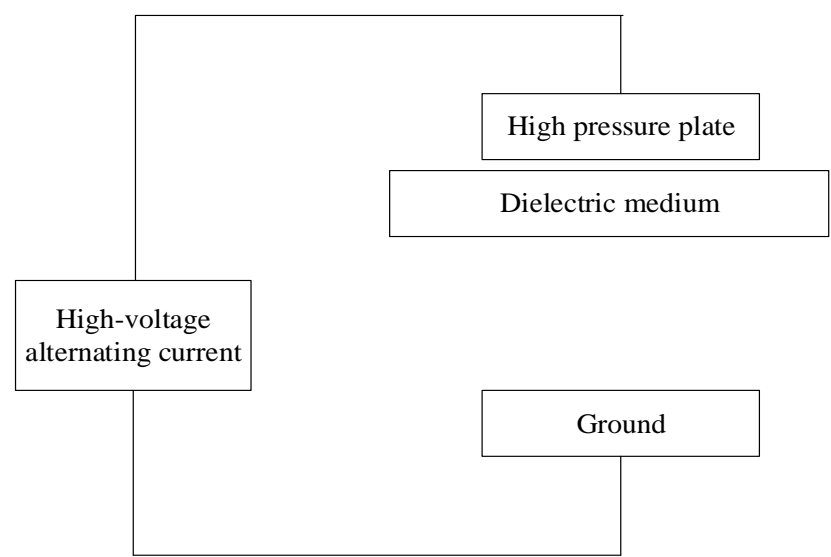

Figure 1. Equivalent diagram of DBD device

Dielectric barrier discharge (DBD) treatment of waste gas in the process of work, the energy transfer of the plasma is generally as follows:

(1) electric field + electron $\rightarrow$ high energy electron

(2) high energy electron + molecular (or atomic) $\rightarrow$ (excited atom, excited group, free radical)

(3) active group + molecule (atom) $\rightarrow$ product + heat

(4) active group + active group $\rightarrow$ product + heat

In the above process, the first electronic energy from the electric field to transfer energy to the atoms or molecules to obtain the excitation or ionization, molecular or atomic energy is excited, while some molecules are ionized, and becoming active groups[6]; these active groups and molecules or atoms collide between active group and active group after the formation of stable products and heat.

Design of the Whole Device. At present, dielectric barrier discharge (DBD) air gap is mainly zero a few millimeters or about a millimeter, this experiment the discharge gap increased to $5 \mathrm{~mm}$, which need high load voltage, increased from several thousand volts to tens of thousands of volts of high voltage power supply. The overall framework is shown in Fig. 2. The introduction of $220 \mathrm{~V}$ city power, after the full wave rectifier, filter to the full bridge inverter, the output of high frequency voltage after the high frequency transformer to load into the load side.

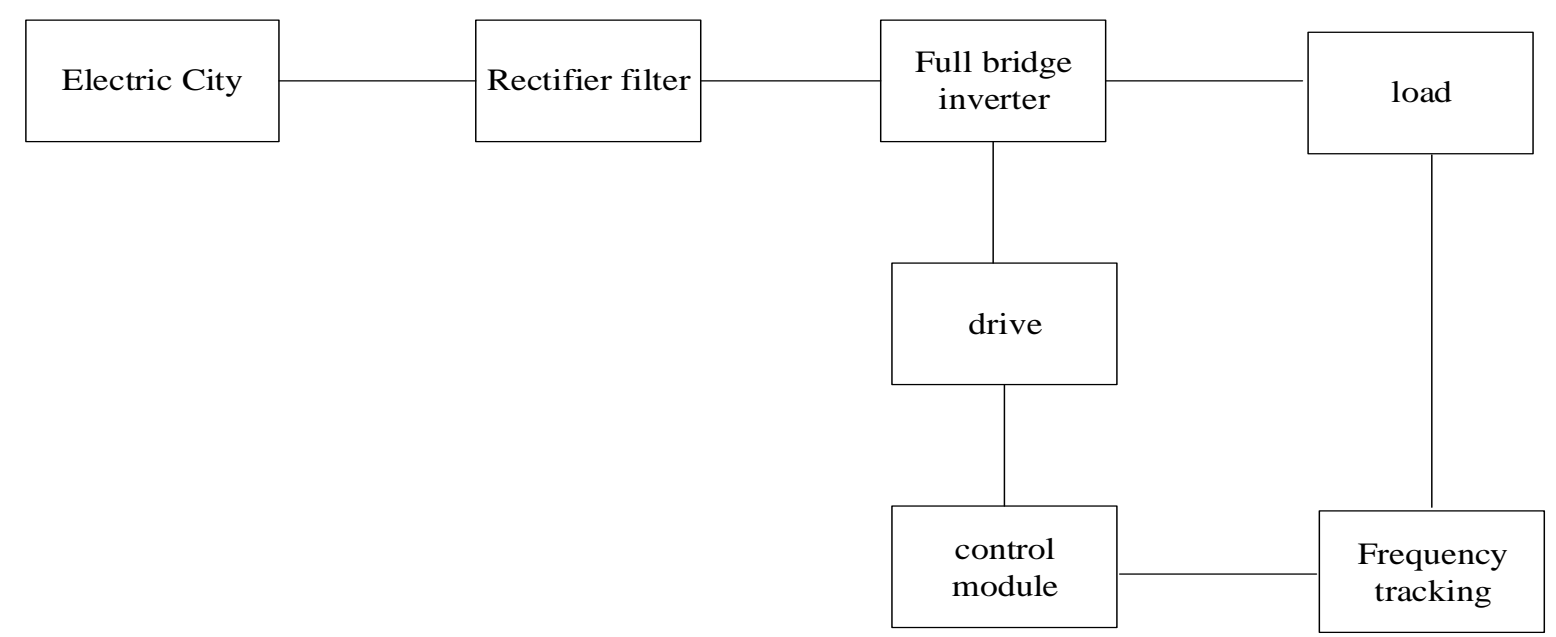

Figure 2. Whole frame of DBD device

The Working Principle of the Inverse Power Transfer. The working process of the inverter is shown in Fig. 3. The switch tube is switched on and off, and the DC voltage is chopped into a 
rectangular wave with positive and negative alternating current. In order to prevent the opening of the switch tube at the same time to burn the switch tube [7], you need to set aside the dead time, the waveform is shown in Fig. 4. The specific process is: Open M2M3, M1M4 close, $U=U_{D}$; close M1M4, open M2M3, U=- $U_{D}$; When switching between M1M4 and M3 M2 at a set frequency, Positive and negative alternating voltages are obtained on the load, the cycle is $1 / \mathrm{f}_{\mathrm{s}}$, This is the working process of the inverter.

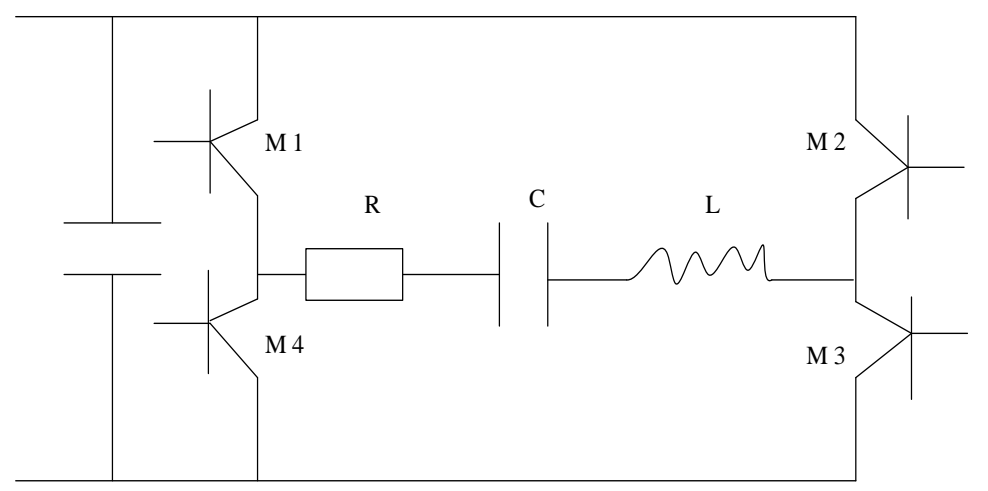

Figure 3. Series resonant equivalent diagram

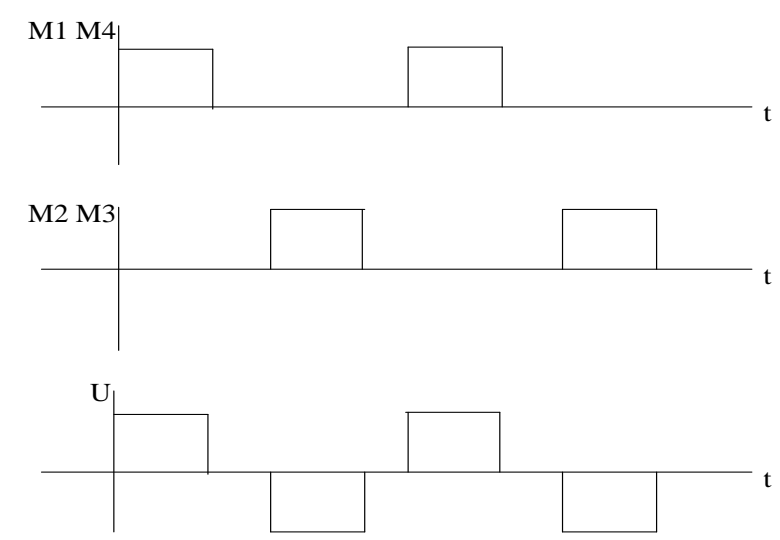

Figure 4. An ideal waveform of the inverter work

The control chip is SG3525, it is a kind of PWM control chip with excellent performance and complete function, It is simple and reliable, and it is very flexible and convenient to use. The output driver for the push-pull output form, increase the driving capability; contains internal under voltage lockout circuit, soft start control circuit, PWM latch and the over-current protection function, frequency adjustable, and can limit the maximum duty ratio. The shock frequency of SG3525 is determined by the external resistance capacitance. The starting frequency of SG3525 is $f_{1}$,

$$
f_{1}=\frac{1}{C_{T}\left(0.67 R_{T}+1.3 R_{D}\right)}
$$

By adjusting the turn-on time to adjust the duty cycle to control the power output of inverter, power adjustment process is as follows: The sampling voltage is delivered to the reverse side of the SG3525 error amplifier and compared with the reference voltage at the same direction. When the sampling voltage is greater than the reference voltage, the duty ratio becomes smaller, the output power decreases; when the sampling voltage is less than the reference voltage, the duty ratio increases, the output power increases. The whole process of work is carried out so that the power of automatic regulation.

Frequency Tracking Technique. With the change of the external environment, such as environmental temperature, humidity and exhaust gas treatment concentration, dielectric barrier discharge (DBD) device characteristics will be changed obviously [8], and the change is nonlinear. 
This will lead to the power supply cannot be good matching of the discharge device, resulting in lower output power and the loss is large. The effective way is to make the switching frequency of power supply always follow the resonant frequency of the equivalent load. This can control the voltage and current load end of the synchronization, and also can reduce switching losses, easy to realize soft switching of power tube [9], so as to reduce the loss and improve the efficiency of. SG3525 pin 3 has an external sync feature, when the synchronous pulse width less than 6us and greater than $0.5 \mathrm{us}$, the frequency of the oscillation frequency is greater than the set [10], the chip will follow the external synchronization pulse oscillation, and two frequency after the output pulse.

\section{Experimental Result Analysis}

Fig. 5 Schematic diagram of the experimental set-up is shown, the harmful gas is collected in the factory of sulfide nitride and other harmful gases, the harmful gas and air are mixed in the gas mixing vessel and are fully mixed into the DBD reactor, set up every minute of the gas flow value, and adjust power supply, collection of samples in the exhaust gas discharged from the back, through the analysis of the sample gas, to adjust the power supply, until the exhaust gas all harmless gas so far. The experimental data is shown in Table 1:

Table 1 Pass into the gas and load power consumption

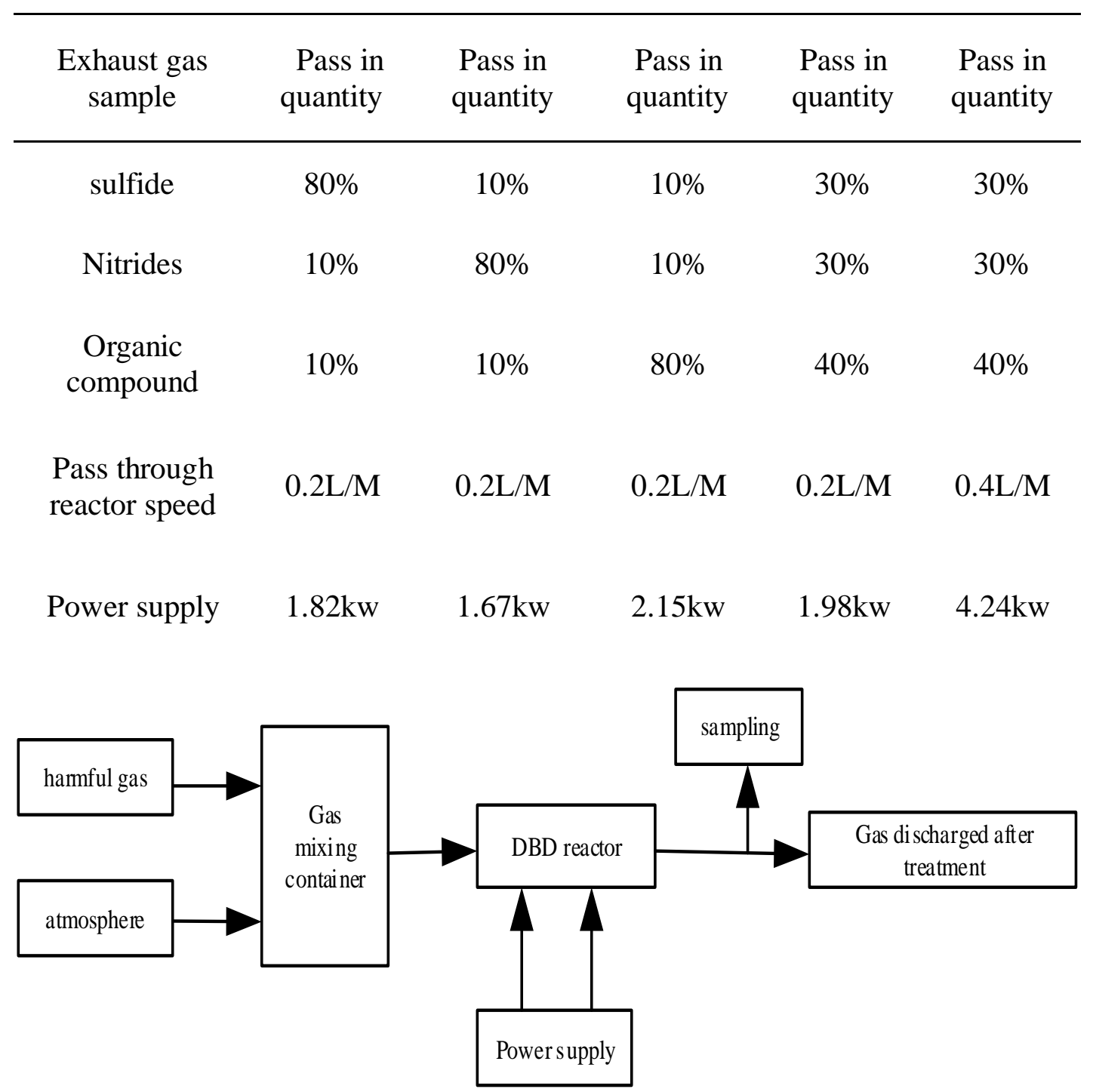

Figure 5. Schematic diagram of experimental device

Through a large number of experiments, collecting waveforms, the waveform of the load side is 
shown in Fig. 6.

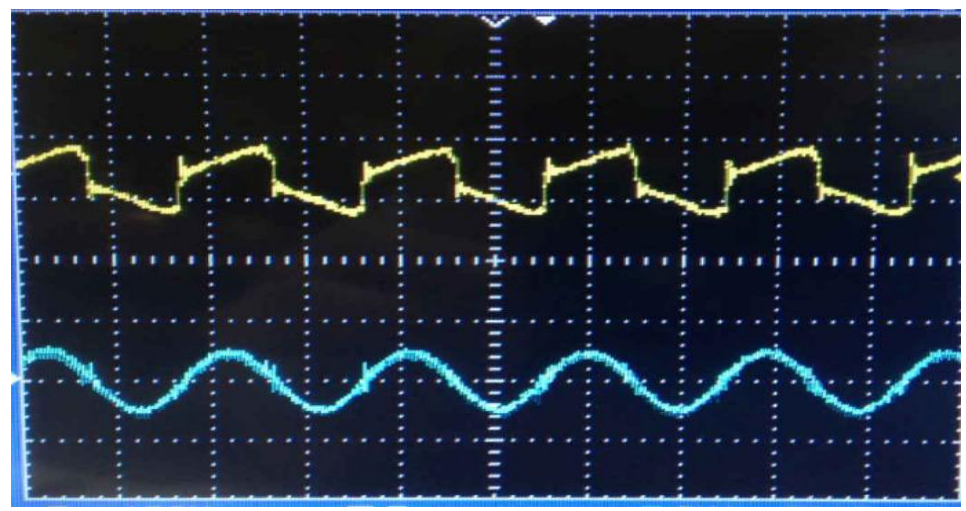

Figure 6. Waveform diagram of the load

As shown in the figure, the inverter output and the load end can be well matched, the output voltage is basically in accordance with the rectangular wave change, load AC current can basically be in accordance with the predetermined sine wave, basically no phase difference.

\section{Summary}

The load will change with the change of the environment, the resonance frequency will change, the need to use frequency tracking technology, so that the frequency of the switch tube is very good matching, tracking the resonant frequency of the load.

Through a lot of experiments and waveform analysis, different working conditions, such as the large difference in the gas concentration, different matching power and frequency should be set up, otherwise the device will not work or the efficiency will be low.

\section{References}

[1] Jin Youmin, three. The physical basis of the low temperature plasma physics [M]. Beijing: Tsinghua University press, 1983.21-30.

[2] Wu Jun, Liu Ping. Design of full bridge switching power supply based on [D] UC3842, Zhengzhou University, 2012, 07:12-16.

[3] Zhang Zhansong, Cai Xuansan. Principle and design of switching power supply (Revised Edition) [M]. Beijing: Publishing House of electronics industry, 2004:288-301.

[4] Chu Bin.IR2110 power driver integrated chip English [A]. Electronics engineer, 2001,30 (10):38-39.

[5] Liu Diantong, Yu Siyuan Liu Jinhua. Frequency tracking control, processing power and constant amplitude control [J]. electromachining \& mould, 2000, (2):68-73.

[6] Li Minyuan, Jiang Haipeng, et al. Selection of optimal dead zone of series resonant inverter [A]. Power electronics technology, 20003, 37 (4):23-28.

[7] Liu Tao.MOSFET series resonant inverter control circuit [A]. Science of the study, 2001 (26):56-59.

[8] Li Chunju, Hou Antonio. High frequency inverter power supply design of [N]. SG3525A and M57959 Engineering Journal of Hunan based on 2008, 18 (4):21-23.

[9] Zhang Zhitao, Zhao Yanhui, Dong Kebing, et al. Study on the resonance of dielectric barrier discharge system [J], high voltage technology, 2004,30 (4): 40-41.

[10] Shao Jianshe, Yan Ping. Resonance phenomenon of dielectric barrier discharge power supply resonant inverter [J], high voltage electrical, 2006, 32 (5): 329-330. 